\title{
MÉTODO ALTERNATIVO PARA A REDUÇÃO DO GRAU DOS POLINÔMIOS PALINDRÔMICOS COM COEFICIENTES REAIS
}

\author{
Duelci A. de Freitas Vaz \\ Instituto Federal de Goiás, Campus Goiânia e PUC Goiás \\ duelci.vaz@ifg.edu.br \\ Julio César Saavedra Vásquez \\ Instituto Federal de Goiás, Campus Goiânia \\ julio.vasquez@ifg.edu.br \\ Regina Célia Bueno da Fonseca \\ Instituto Federal de Goiás, Campus Goiânia e IF-UnB, Campus Darcy Ribeiro \\ regina.fonseca@ifg.edu.br
}

\section{Resumo}

O comportamento dos zeros dos polinômios algébricos é uma das subáreas clássicas da Análise. Tal assunto é muito abordado na Matemática e atualmente as funções polinomiais são temas de muita investigação, tanto na área computacional, quanto na teórica. O comportamento dos zeros de polinômios palindrômicos no círculo unitário é motivo de pesquisa na literatura [1], [2] e [3]. Nos polinômios palindrômicos $P_{2 n}(z)$, $n$ inteiro, de grau par, sabe-se que podem ser expressos por $P_{2 n}(z)=z^{n} Q_{n}(z+1 / z)$, onde $Q_{n}$ é um polinômio de grau $n$, de tal forma que a partir das raízes de $Q_{n}$, obtemos as raízes de $P_{2 n}(z)$. Conrad [4] aborda um procedimento para tal propósito. Neste trabalho apresentamos um método alternativo com a mesma finalidade.

Palavras chaves: redução de grau; polinômios palindrômicos; coeficientes reais.

\section{Abstract}

The behavior of the zeros of the algebraic polynomials is one of the classic subareas of Analysis. This subject is widely discussed in Mathematics and currently polynomial functions are subjects of much investigation, both in the computational and theoretical areas. The behavior of the zeros of palindromic polynomials in the unit circle is a reason for research in the literature [1], [2] and [3]. In palindromic polynomials $P_{2 n}(z)$, $n$ interger, of even degree, it is known that they can be expressed by $P_{2 n}(z)=z^{n} Q_{n}(z+$ $1 / z)$, where $Q_{n}$ is a polynomial of degree $n$, such that from the roots of $Q_{n}$, we obtain the roots of $P_{2 n}(z)$. Conrad [4] addresses a procedure for this purpose. In this work, we present an alternative method with the same purpose.

Keywords: degree reduction; palindromic polynomials; real coefficients. 
Vaz, D. A. F.; Vásquez, J. C. S.; Fonseca, R. C. B.

\section{Introdução}

Da literatura, sabe-se que dado um polinômio $P(z)=\sum_{k=0}^{n} a_{k} z^{k}, a_{k} \in \mathbb{R}$, as seguintes condições são equivalentes:

i. $P(z)$ é palindrômico, isto é, $a_{n-k}=a_{k}, \forall k=0,1,2, \ldots, n$.

ii. $P(z)=z^{n} P\left(\frac{1}{z}\right)$. Segue-se que se $z_{0}$ é uma raiz de $P, \overline{z_{0}}, \frac{1}{z_{0}}$ e $\frac{1}{z_{0}}$ também o serão. Caso $\left|z_{0}\right|<1$, então $z_{0}$ e $\overline{z_{0}}$ estarão localizadas simetricamente no interior do disco $|z|<1$ e $\frac{1}{z_{0}}$ e $\frac{1}{z_{0}}$ fora deste. Logo se $\left|z_{0}\right|=1$, teremos apenas outra raiz complexa para $P(z)$, uma vez que $\overline{z_{0}}=\frac{1}{z_{0}}$.

iii. No caso de $n$ ser par, $P_{n}(z)=z^{\frac{n}{2}} Q_{n}\left(z+\frac{1}{z}\right)$, para um polinômio $Q_{n}$ de grau $\frac{n}{2}$.

Do item iii., segue-se que todo polinômio palindrômico $P_{n}(z)$, de grau $n$ par, pode ser expresso em termos de $w=z+\frac{1}{z}$ e que cada raiz $w$ de $Q_{n}$ gera duas raízes de $P_{n}(z)$. Observe-se que no caso de um polinômio palindrômico $P(z)$ de grau ímpar temse: $P_{2 n+1}(z)=(z+1) z^{n} Q_{n}\left(z+\frac{1}{z}\right)$, uma vez que $z=-1$ é raiz de todo polinômio palíndromo de grau ímpar. Logo, sem perda de generalidade é possível supor que $P_{n}(z)$ é de grau par.

Conrad [4] efetua várias manipulações algébricas no polinômio do exemplo abaixo para achar $Q_{n}$ e consequentemente as raízes de $P(z)$. De fato, o polinômio palindrômico

$$
P(z)=z^{8}-z^{5}-z^{4}-z^{3}+1=z^{4}\left(z^{4}-z-1-\frac{1}{z}+\frac{1}{z^{4}}\right)
$$

desta forma,

$$
\left(z^{4}-z-1-\frac{1}{z}+\frac{1}{z^{4}}\right)-\left(z+\frac{1}{z}\right)^{4}=-4 z^{2}-z-7-\frac{1}{z}-\frac{4}{z^{2}} .
$$

Adicionando o termo $4\left(z+\frac{1}{z}\right)^{2}$ na equação $(1.2)$, temos

$$
z^{4}\left(z^{4}-z-1-\frac{1}{z}+\frac{1}{z^{4}}\right)-\left(z+\frac{1}{z}\right)^{4}+4\left(z+\frac{1}{z}\right)^{2}=-z+1-\frac{1}{z} .
$$

Adicionando o termo $\left(z+\frac{1}{z}\right)$ nos dois lados da igualdade na equação (1.3), temos:

$$
\left(z^{4}-z-1-\frac{1}{z}+\frac{1}{z^{4}}\right)-\left(z+\frac{1}{z}\right)^{4}+4\left(z+\frac{1}{z}\right)^{2}+\left(z+\frac{1}{z}\right)=1
$$


Vaz, D. A. F.; Vásquez, J. C. S.; Fonseca, R. C. B.

Multiplicando o termo $z^{4}$ nos dois lados da igualdade na equação (1.4), temos:

$$
z^{4}\left(z^{4}-z-1-\frac{1}{z}+\frac{1}{z^{4}}\right)=z^{4}\left(\left(z+\frac{1}{z}\right)^{4}-4\left(z+\frac{1}{z}\right)^{2}-\left(z+\frac{1}{z}\right)+1\right) .
$$

Desta forma, da equação (1.5), obtemos

$$
P_{8}(z)=z^{4} Q_{4}\left(z+\frac{1}{z}\right)
$$

Conforme descrito no item iii., a equação (1.6) é um polinômio palindrômico de grau $\operatorname{par}(n=8)$.

Observação 1.1. Observamos que este procedimento desenvolvido acima envolve várias manipulações algébricas as quais dependem integralmente das expressões que as precedem.

Assim, na Seção 2 mostramos um processo alternativo de redução de polinômio palindrômico com coeficientes reais.

\section{Processo alternativo de redução do polinômio pa- lindrômico com coeficientes reais}

O procedimento a seguir que tem como base as relações de Viète ${ }^{\mathrm{A}}$ permite de maneira alternativa e genericamente expressar:

$$
P_{2 n}(z)=z^{n} Q_{n}\left(z+\frac{1}{z}\right)
$$

em que $a_{n} \neq 0$. Sem perda de generalidade, considere o polinômio palindrômico de grau par:

$$
P_{2 n}(z)=z^{2 n}+a_{1} z^{2 n-1}+a_{2} z^{2 n-2}+\cdots+a_{2} z^{2}+a_{1} z+1 .
$$

Das relações de Viète, tem-se que, se $z_{i}(i=1,2, \cdots, 2 n)$ são as raízes de $P_{2 n}(z)$, então:

$$
\sum_{i=1}^{2 n} z_{i}=-a_{1}, \sum_{j \neq i}^{n} z_{i} z_{j}=a_{2}, \sum_{i \neq j \neq k}^{2 n} z_{i} z_{j} z_{k}=-a_{3}, \cdots, z_{1} z_{2} z_{3} \cdots z_{2 n-1} z_{2 n}=1
$$

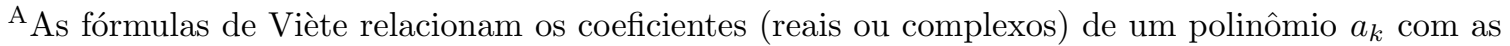
somas e produtos de suas raízes, denominadas por François Viète [6].
}

ReviSeM, Ano 2021, $N^{\circ}$. 3, 1-11 
Uma vez que estas raízes ocorrem em pares inversos $\left(z_{2 i-1}, z_{2 i}\right)$, onde $z_{2 i}=\frac{1}{z_{2 i-1}}$, $(i=1,2, \cdots, n)$, a partir da mudança de variável $x_{i}=z_{2 i-1}+z_{2 i}, z_{2 i}=\frac{1}{z_{2 i-1}}$, $i=1,2, \cdots, n$, é possível construir o polinômio $Q_{n}(x)$ :

$$
Q_{n}\left(z+\frac{1}{z}\right)=Q_{n}(x)=x^{n}+b_{1} x^{n-1}+b_{2} x^{n-2}+\cdots+b_{n-2} x^{2}+b_{n-1} x+b_{n},
$$

onde os coeficientes $b_{k},(k=1,2, \cdots, n)$ são obtidos a partir das relações das raízes de $P_{n}(z)$ e das relações com seus coeficientes, além do fato que $z_{2 i-1} z_{2 i}=1, i=1,2, \cdots, n$. De posse do polinômio $Q_{n}(x)$ e a partir de suas raízes $x_{i}(i=1,2, \cdots, n)$, obtemos as $2 n$ raízes de $P_{n}(z)$, decorrentes da solução das $n$ equações quadráticas:

$$
x_{i}=z_{2 i-1}+z_{2 i}=z_{2 i-1}+\frac{1}{z_{2 i-1}}, \quad i=1,2, \cdots, n .
$$

De fato, se $Q_{n}(x)=x^{n}+b_{1} x^{n-1}+b_{2} x^{n-2}+\cdots+b_{n-2} x^{2}+b_{n-1} x+b_{n}$, temos que:

$$
\begin{gathered}
-b_{1}=\sum_{i=1}^{n} x_{i}=\sum_{i=1}^{n}\left(z_{2 i-1}+z_{2 i}\right)=\sum_{i=1}^{2 n} z_{i}=-a_{1}, \\
b_{2}=\sum_{i \neq j}^{n} x_{i} x_{j}=\sum_{i \neq j}^{n}\left(z_{2 i-1}+z_{2 i}\right)\left(z_{2 j-1}+z_{2 j}\right)=\sum_{i \neq j}^{2 n} z_{i} z_{j}-\sum_{i=1}^{2 n} z_{i} z_{j}=a_{2}-n .
\end{gathered}
$$

Na equação (2.7) podemos analisar cada um de seus termos relacionando-os com cada termo da equação (2.3): uma vez que $\sum_{i \neq j}^{2 n} z_{i} z_{j}$ contém termos do tipo $z_{2 i-1} z_{2 i}=1$, que não estão presentes no desenvolvimento de $\sum_{i \neq j}^{n}\left(z_{2 i-1}+z_{2 i}\right)\left(z_{2 j-1}+z_{2 j}\right)$, o número excedente deste tipo de parcelas de valor 1 é dado por

$$
C_{2}^{2 n}-2^{2} C_{2}^{n}=n .
$$

Por outro lado,

$$
-b_{3}=\sum_{i \neq j \neq k}^{n} x_{i} x_{j} x_{k}=\sum_{i \neq j \neq k}^{n}\left(z_{2 i-1}+z_{2 i}\right)\left(z_{2 j-1}+z_{2 j}\right)\left(z_{2 k-1}+z_{2 k}\right) .
$$

Precisamos escrever o desenvolvimento da equação (2.9) em função de $z_{i} z_{j} z_{k}, i \neq j \neq k$, de fato,

$$
-b_{3}=\sum_{i \neq j \neq k} z_{i} z_{j} z_{k}-\sum z_{2 i-1} z_{2 i} z_{k}
$$


Pois ternas do tipo $z_{2 i-1} z_{2 i} z_{k}=z_{k}$ não estão presentes nas $2^{3} C_{3}^{n}$ parcelas do desenvolvimento de (2.9), o número excedente deste tipo de parcelas é dado por: $C_{3}^{2 n}-2^{3} C_{3}^{n}=$ $2 n(n-1)$, pois $\sum_{i \neq j \neq k} z_{i} z_{j} z_{k}$ tem $C_{3}^{2 n}$ ternas. Portanto, a equação (2.10) pode ser reescrita:

$$
-b_{3}=\sum_{i \neq j \neq k}^{2 n} z_{i} z_{j} z_{k}-(n-1) \sum_{k=1}^{2 n} z_{2 i-1} z_{2 i} z_{k}
$$

Usando as relações de (2.3) na equação (2.11), obtemos

$$
\begin{aligned}
-b_{3} & =-a_{3}-(n-1) \sum_{k=1}^{2 n} z_{k}=-a_{3}-(n-1)\left(-a_{1}\right) \\
b_{3} & =a_{3}-(n-1) a_{1} .
\end{aligned}
$$

Agora vamos ao cálculo de:

$$
b_{4}=\sum_{i \neq j \neq k \neq l}^{n} x_{i} x_{j} x_{k} x_{l}=\sum_{i \neq j \neq k \neq l}^{n}\left(z_{2 i-1}+z_{2 i}\right)\left(z_{2 j-1}+z_{2 j}\right)\left(z_{2 k-1}+z_{2 k}\right)\left(z_{2 l-1}+z_{2 l}\right) .
$$

Observe que esta última expressão, o seu desenvolvimento contém $2^{4} C_{4}^{n}$ parcelas. Uma vez que parcelas do tipo $z_{2 i-1} z_{2 i} z_{j} z_{l}=z_{j} z_{l} \neq 1$ e $z_{2 i-1} z_{2 i} z_{2 j-1} z_{2 j}=1$, não estão presentes. Dessa forma, se substituirmos $\sum_{i \neq j \neq k \neq l}^{2 n} z_{i} z_{j} z_{k} z_{l}$ na expressão que determina $b_{4}$, teremos:

$$
b_{4}=\sum_{i \neq j \neq k \neq l} z_{i} z_{j} z_{k} z_{l}-\left(\sum_{z_{j} z_{l} \neq 1} z_{2 i-1} z_{2 i} z_{j} z_{l}+\sum z_{2 i-1} z_{2 i} z_{2 j-1} z_{2 j}\right) .
$$

O total excedente destes dois tipos de parcelas é dado por:

$$
C_{4}^{2 n}-2^{4} C_{4}^{n}=\frac{n(n-1)(4 n-7)}{2}
$$

Das quais $C_{2}^{n}$ parcelas são do tipo $z_{2 i-1} z_{2 i} z_{2 j-1} z_{2 j}=1$, logo:

$$
b_{4}=\sum_{i \neq j \neq k \neq l}^{2 n} z_{i} z_{j} z_{k} z_{l}-\left(\sum_{z_{j} z_{l} \neq 1} z_{j} z_{l}+\frac{n(n-1)}{2}\right) .
$$

Por outro lado, o total de parcelas do tipo $z_{2 i-1} z_{2 i} z_{j} z_{l}=z_{j} z_{l} \neq 1$ é

$$
C_{4}^{2 n}-2^{4} C_{4}^{n}-C_{2}^{n}=2 n(n-1)(n-2) .
$$


Vaz, D. A. F.; Vásquez, J. C. S.; Fonseca, R. C. B.

Uma vez que $\sum_{j \neq l}^{2 n} z_{j} z_{l}$ contém $C_{2}^{2 n}$ parcelas, das quais $n$ são do tipo $z_{2 i-1} z_{2 i}=1 \mathrm{e}$ $C_{2}^{2 n}-n=2 n(n-1)$ do tipo $z_{j} z_{l} \neq 1$, segue-se que $\sum_{z_{j} z_{l} \neq 1} z_{j} z_{l}$ contém exatamente $(n-2)$ vezes a quantidade deste tipo de termos contidos em $\sum_{k \neq l}^{2 n} z_{k} z_{l}$, portanto:

$$
\sum_{z_{j} z_{l} \neq 1} z_{j} z_{l}=(n-2)\left(\sum_{k \neq l}^{2 n} z_{k} z_{l}-n\right)
$$

Substituindo esta última relação (2.18) na equação (2.16), temos:

$$
b_{4}=\sum_{i \neq j \neq k \neq l}^{2 n} z_{i} z_{j} z_{k} z_{l}-\left((n-2) \sum_{k \neq j}^{2 n} z_{k} z_{l}-\frac{n(n-3)}{2}\right),
$$

ou equivalentemente,

$$
b_{4}=a_{4}-(n-2) a_{2}+\frac{n(n-3)}{2} .
$$

O cálculo do coeficiente $b_{5}$ do polinômio $Q_{n}(x)$ dado na equação (2.4):

$$
\begin{gathered}
-b_{5}=\sum_{i \neq j \neq k \neq l \neq r}^{n} x_{i} x_{j} x_{k} x_{l} x_{r} \\
=\sum_{i \neq j \neq k \neq l \neq r}^{n}\left(z_{2 i-1}+z_{2 i}\right)\left(z_{2 j-1}+z_{2 j}\right)\left(z_{2 k-1}+z_{2 k}\right)\left(z_{2 l-1}+z_{2 l}\right)\left(z_{2 r-1}+z_{2 r}\right) \\
=\sum_{i \neq j \neq k \neq l \neq r}^{2 n} z_{i} z_{j} z_{k} z_{l} z_{r}-\left(\sum z_{2 i-1} z_{2 i} z_{j} z_{k} z_{l}+\sum z_{2 i-1} z_{2 i} z_{2 j-1} z_{2 j} z_{k}\right) \\
=\sum_{i \neq j \neq k \neq l \neq r}^{2 n} z_{i} z_{j} z_{k} z_{l} z_{r}-\left(\sum z_{j} z_{k} z_{l}+\sum z_{k}\right) .
\end{gathered}
$$

Uma vez que a quantidade de parcelas $z_{2 i-1} z_{2 i} z_{2 j-1} z_{2 j} z_{k}=z_{k}$ desta última é $(2 n-4) C_{2}^{n}$, logo o número de termos do $z_{j} z_{k} z_{l}$ será dado por:

$$
C_{2}^{2 n}-2^{2} C_{2}^{n}=n^{\mathrm{B}}
$$

$$
{ }^{\mathrm{B}} C_{n}^{k}=\frac{n !}{k !(n-k) !}, \operatorname{com} k=0,1,2,3, \cdots, n .
$$


Por outro lado, $\sum_{i \neq j \neq k}^{2 n} z_{i} z_{j} z_{k}$ contém $C_{3}^{2 n}$ parcelas, das quais $(2 n-2) C_{1}^{n}$ são do tipo $z_{i} z_{j} z_{k}=z_{k}$ e $C_{3}^{2 n}-2(n-1) C_{1}^{n}=\frac{4 n(n-1)(n-2)}{3}$, termos tal que $z_{i} z_{j} \neq 1$, segue-se que $\sum_{j \neq k \neq l} z_{j} z_{k} z_{l}$ contém exatamente $(n-3)$ vezes a quantidade deste tipo de termos presentes em $\sum_{j \neq k \neq l}^{2 n} z_{j} z_{k} z_{l}$, portanto:

$$
\sum z_{2 i-1} z_{2 i} z_{j} z_{k} z_{l}=(n-3)\left(\sum_{z_{j} z_{l} \neq 1} z_{j} z_{k} z_{l}-\sum z_{k}\right) .
$$

Segue-se que o número de parcelas do tipo $z_{i} z_{j} z_{k}=z_{k}$ é

$$
\left(-(n-3) C_{2}^{n}+2(n-2) C_{2}^{n}\right)=\frac{(n-4)(n-1) n}{2} .
$$

Substituindo estas duas últimas relações em (2.21), obtemos

$$
-b_{5}=\sum_{i \neq j \neq k \neq l \neq r}^{2 n} z_{i} z_{j} z_{k} z_{l} z_{r}-(n-3) \sum z_{j} z_{k} z_{l}+\frac{(n-4)(n-1)}{2} \sum_{k=1}^{2 n} z_{k} .
$$

Usando as relações (2.3) na equação (2.25), obtemos

$$
b_{5}=a_{5}-(n-3) a_{3}+\frac{(n-4)(n-1)}{2} a_{1} .
$$

Pelo exposto acima concluímos que cada coeficiente $b_{k}$ de índice par/ímpar depende de todos os coeficientes $a_{k}$ de índice par/ímpar inferiores ou iguais a $k$. Seguindo um procedimento análogo ao supracitado é possível obter os $b_{k}, k \geq 6$.

Por exemplo, no caso de um polinômios palindrômicos de grau 8 e 6 :

$$
\begin{gathered}
P(z)=z^{8}+a_{1} z^{7}+a_{2} z^{6}+a_{3} z^{5}+a_{4} z^{4}+a_{3} z^{3}+a_{2} z^{2}+a_{1} z+1 \\
P(z)=z^{6}+a_{1} z^{5}+a_{2} z^{4}+a_{3} z^{3}+a_{2} z^{2}+a_{1} z+1,
\end{gathered}
$$

seus correspondentes polinômios reduzidos são respectivamente:

$$
\begin{gathered}
Q_{4}(x)=x^{4}+a_{1} x^{3}+\left(a_{2}-4\right) x^{2}+\left(a_{3}-3 a_{1}\right) x+\left(a_{4}-2 a^{2}+2\right) . \\
Q_{3}(x)=x^{3}+a_{1} x^{2}+\left(a_{2}-3\right) x+\left(a_{3}-2 a_{1}\right) .
\end{gathered}
$$


Assim, por exemplo, no caso específico do polinômio

$$
P_{8}(z)=z^{8}-z^{5}-z^{4}-z^{3}+1,
$$

temos que seu polinômio reduzido é:

$$
Q_{4}(x)=x^{4}-4 x^{2}-x+1,
$$

uma vez que $a_{1}=0, a_{2}-4=-4, a_{3}-3 a_{1}=-1$ e $a_{4}-2 a_{2}+2=1$. Pelas equações (2.1), (2.4) e (2.32), o polinômio (2.31) pode ser exprimido como:

$$
P_{8}(z)=z^{4} Q_{4}\left(z+\frac{1}{z}\right)=z^{4}\left(\left(z+\frac{1}{z}\right)^{4}-4\left(z+\frac{1}{z}\right)^{2}-\left(z+\frac{1}{z}\right)+1 .\right)
$$

A partir da argumentação dada acima podemos enunciar e demonstrar o seguinte teorema.

Teorema 2.1. Considere o polinômio palindrômico de grau par e com coeficientes reais,

$$
P(z)=z^{2 n}+a_{1} z^{2 n-1}+a_{2} z^{2 n-2}+\cdots+a_{2} z^{2}+a_{1} z+1,
$$

e seja $Q_{n}(x)$ seu polinômio reduzido. Então $x$ é uma raiz real de $Q_{n}(x)$ se, e somente, $P(z)$ possui duas raízes $z$, tais que z é real ou $|z|=1$. Ou, equivalente, para cada raiz complexa de $Q_{n}(x), P(z)$ possuirá 2 raízes complexas $z,|z| \neq 1$ e reciprocamente.

Demonstração. Seja $z=\alpha+\beta i$ uma raiz qualquer $P(z) \operatorname{logo}$ as raízes de $Q_{n}(x)$ são tais que

$$
x=z+\frac{1}{z}=z+\frac{\bar{z}}{|z|^{2}}=\alpha\left(1+\frac{1}{|z|^{2}}\right)+\beta\left(1-\frac{1}{|z|^{2}}\right) i .
$$

Logo $x$ será real se, e somente se, $z \in \mathbb{R}$ ou $|z|=1$.

Observa-se que, se $x \in \mathbb{R}$, da equação (2.35) tem-se

$$
z=\frac{-x \pm \sqrt{x^{2}-4}}{2}
$$

$\log 0$

$$
z \in \mathbb{C} \backslash \mathbb{R} \Leftrightarrow x \in(-2,2) .
$$

Além disso, $|z|=\sqrt{\left(-\frac{x}{2}\right)^{2}+\left(\frac{\sqrt{4-x^{2}}}{2}\right)^{2}}=1^{\mathrm{C}}$. Reciprocamente, $\mathrm{s} x \in \mathbb{R}$,

$$
|x| \geq 2 \Leftrightarrow z \in \mathbb{R}
$$

de tal sorte que $z$ e $1 / z$ (diferente $+/-1$ ) não estão no círculo unitário.

\footnotetext{
${ }^{\mathrm{C}}$ De fato, da equação $(2.36)$ temos: $z=-\frac{x \pm \sqrt{-\left(4-x^{2}\right)}}{2} \quad \Longrightarrow \quad z=-\frac{x}{2} \pm i \frac{\sqrt{\left(4-x^{2}\right)}}{2} \Longrightarrow$ $|z|=1$.
} 
Exemplo 2.2. Considere o polinômio reduzido

$$
Q_{5}(x)=x^{5}+x^{4}-6 x^{3}-3 x^{2}+7 x+5
$$

do polinômio

$$
P(z)=z^{10}+z^{9}-z^{8}+z^{7}-z^{6}+5 z^{5}-z^{4}+z^{3}-z^{2}+z+1
$$

A figura 1 mostra as raízes (ou zeros) $z_{i}, i=1,2, \cdots 10$ do polinômio $P(z)$ dado na equação (2.38) e suas localizações no círculo unitário.

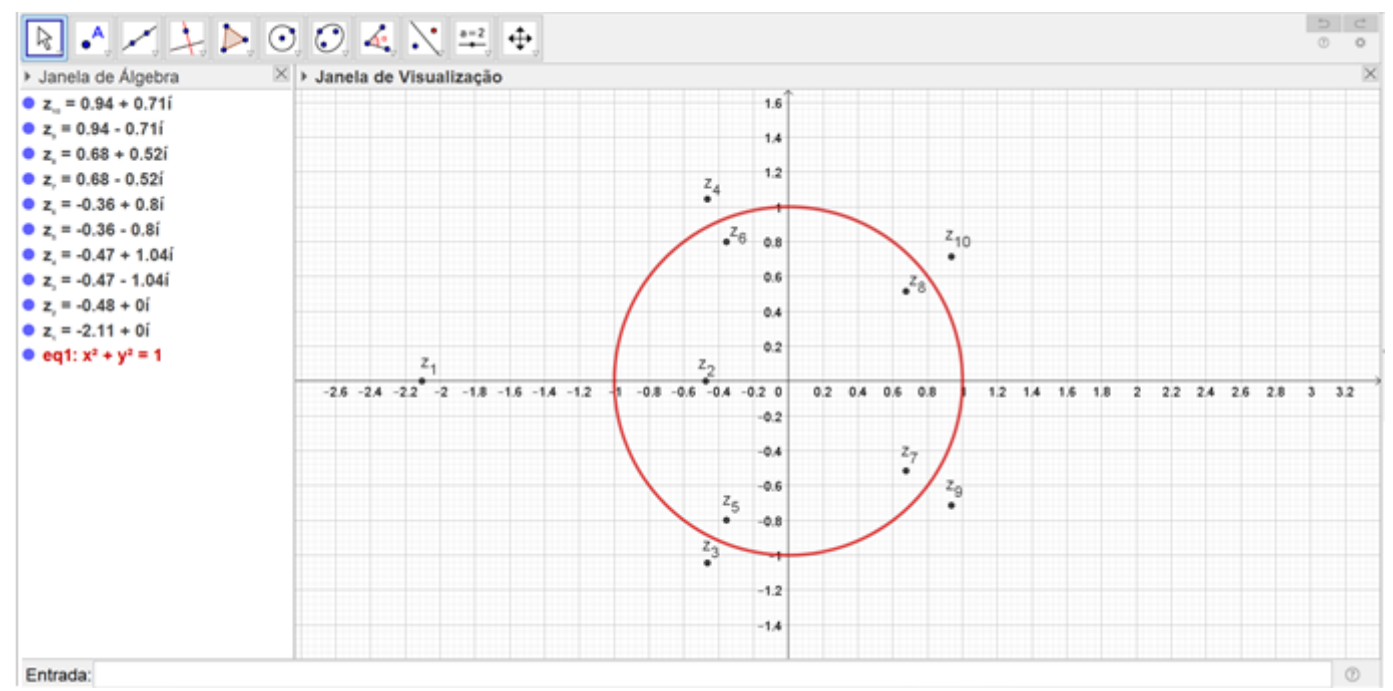

Figura 1: Raízes do polinômio $P(z)$ dado na equação (2.38) no círculo unitário.

A partir das 5 raízes $x_{i}$ de $Q_{n}(x)$ dada na equação (2.37), resolvemos as 5 correspondentes equações quadráticas para achar as 10 raízes de $P(z)$ dada na equação $(2.38)$. Por exemplo, para $x=-2,58$ da equação (2.5), temos

$$
-2,58=z+\frac{1}{z}
$$

Na figura 1 podemos observar as duas soluções da equação (2.39): $z=-0,48$ e $z=-2,1$ que correspondem às duas raízes de $P(z)$ da equação (2.38).

Exemplo 2.3. Seja o polinômio

$$
P(z)=z^{9}+4 z^{8}+3 z^{7}+2 z^{6}+z^{5}+z^{4}+2 z^{3}+3 z^{2}+4 z+1 .
$$


Uma vez que o polinômio dado na equação (2.40) pode ser escrito como

$$
P(z)=(z+1)\left(z^{8}+3 z^{7}+2 z^{5}-z^{4}+2 z^{3}+3 z+1\right),
$$

segue que o polinômio reduzido de $z^{8}+3 z^{7}+2 z^{5}-z^{4}+2 z^{3}+3 z+1$ é

$$
Q_{4}(x)=x^{4}+3 x^{3}-2 x^{2}-10 x .
$$

A figura 2 mostra as raízes (ou zeros) $z_{i}, i=1,2, \cdots 9$ do polinômio $P(z)$ dado na equação (2.40) e suas localizações no círculo unitário.

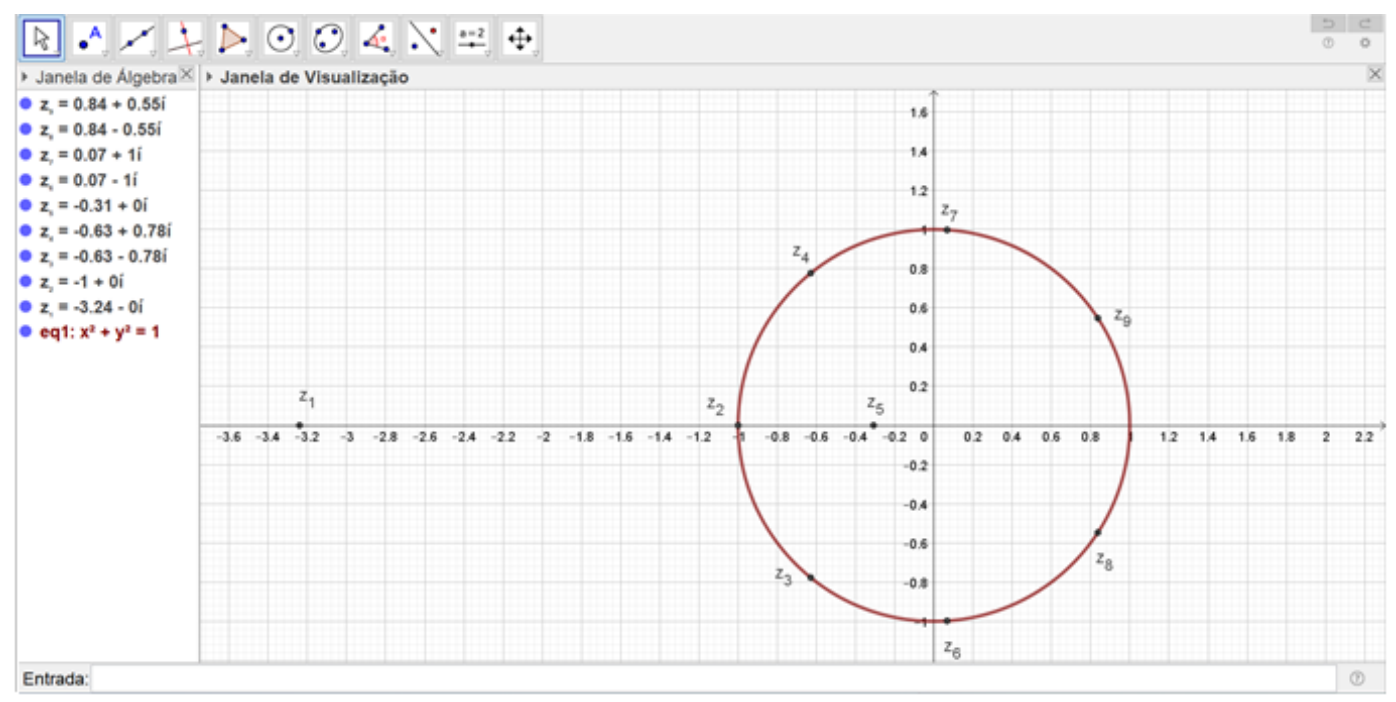

Figura 2: Raízes do polinômio $P(z)$ dado na equação (2.40) no círculo unitário.

\section{Resultados Principais}

É interessante observar como a geometria intrínseca das raízes dos polinômios palindrômicos simplifica o cálculo das mesmas. Temos apresentado aqui um processo recursivo que permite a redução do grau de um polinômio palindrômico facilitando o cálculo bem como a determinação da natureza e alocação das suas raízes em relação ao círculo unitário. Por outro lado, se impormos condições nos coeficientes $b_{k}$ de $Q_{n}(x)$ que definam a natureza das suas raízes e posição em relação a $|z|=1$ isto permitirá construirmos seu correspondente polinômio palindrômico cujas raízes terão natureza e alocação decorrente das raízes de $Q_{n}(x)$ verificando assim as condições constantes nos trabalhos de [2], [3] e [5]. 
Vaz, D. A. F.; Vásquez, J. C. S.; Fonseca, R. C. B.

\section{Referências}

[1] Kwon, Do Yong. Reciprocal polynomials with all zeros on the unit circle. Acta Math. Hung v. 131(3), p. 285-294, 2011. https://doi.org/10.1007/ s10474-011-0090-6

[2] Lakatos, Piroska. On zeros of reciprocal polynomials. Publ. Math. Debrecen, v. 61(3-4), p. 645-661, 2002. ISSN 0033-3883

[3] Marden, Morris. Geometry of polynomials. Mathematical Surveys and Monographs, Vol. 3, American Mathematicals Society, 2nd edition, Providence, Rhode Island, 1966. https://dx.doi.org/10.1090/surv/003

[4] Conrad, Keith. Symmetric Polynomials. Kconrad.math.uconn.edu editor in the Ring Theory. Expository papers, unpublished notes available at https:// kconrad.math.uconn.edu/blurbs/ - Ver na página de notas/arquivos das exposições (do autor) e descer até o bloco "Teoria de Anel", onde encontrará a referência "Polinômios Simétricos". Postado Online e acessado em 05-Junho-2020.

[5] Meneguette, Messias. Zeros in the unit disk. SIAM Review, v. 36(4), pp. 656-657, 1994. https://doi.org/10.1137/1036150

[6] Viète, François. The Analytic Art: Nine Studies in Algebra, Geometry and Trigonometry from the Opus Restitutae Mathematicae Analyseos, seu Algebrâ Novâ. Dover Publications Inc. [Unabridged republication of the work originally published by The Kent State University Press, Kent, Ohio, 1983.] Mineola, New York: 2006. ISBN 0-486-45348-0

Submetido em 01 de Agosto de 2020. $1^{\text {a }}$ Decisão em 30 de Setembro de 2020. Aceito em 04 de Junho de 2021. 